\title{
Pediatric Multiple Endocrine Neoplasia Type 2B: Clinicopathological Correlation of Perilimbal Mucosal Neuromas and Treatment of Secondary Open-Angle Glaucoma
}

\author{
Ta Chen Chang Kingsley C. Okafor Kara M. Cavuoto Sander R. Dubovy Carol L. Karp \\ Bascom Palmer Eye Institute, University of Miami Miller School of Medicine, Miami, FL, USA
}

\section{Established Facts}

- Mucosal neuromas are associated with multiple endocrine neoplasia type 2B (MEN 2B), the histopathology of which is well described.

- Open-angle glaucoma can rarely occur with MEN 2B.

- Surgical treatment approach of MEN 2B-associated glaucoma is not well established.

\section{Novel Insights}

- High-resolution anterior segment optical coherence tomographic findings of mucosal neuroma can help in its diagnosis.

- Gonioscopy-assisted transluminal trabeculotomy may be safe and effective in the treatment of juvenile-onset open-angle glaucoma associated with MEN 2B.

\section{Keywords}

Multiple endocrine neoplasia type $2 \mathrm{~B} \cdot$ Mucosal neuromas · Juvenile open-angle glaucoma · Anterior segment optical coherence tomography - Gonioscopy-assisted transluminal trabeculotomy

\section{Abstract}

Ocular findings of multiple endocrine neoplasia type 2B (MEN $2 B$ ) include prominent corneal nerves, mucosal neuromas of the conjunctiva, glaucoma, and dry eyes. A 15-year-old girl with MEN 2B presents with advanced secondary open-angle glaucoma and bilateral perilimbal masses. High-resolution optical coherence tomography (HR-OCT) of the perilimbal lesions showed normal epithelial thickness and subepithelial lobular areas of mixed reflectivity, which correlates well with histo- pathologic findings of benign mucosal neuromas. The patient underwent bilateral gonioscopy-assisted transluminal trabeculotomy (GATT) in both eyes, which achieved adequate intraocular pressure control at short-term follow-up. To the authors' knowledge, this is the first report correlating HR-OCT and histopathologic findings in benign mucosal neuroma associated with MEN 2B, and the first description of secondary open-angle glaucoma associated with MEN 2B treated with GATT.

(c) 2017 S. Karger AG, Basel

\section{Introduction}

Ocular manifestations of multiple endocrine neoplasia type 2B (MEN 2B), a rare, autosomal-dominant tumor syndrome, include prominent corneal nerves, mucosal

\section{KARGER}

(c) 2017 S. Karger AG, Basel

E-Mail karger@karger.com

www.karger.com/oop
Ta Chen Chang

Bascom Palmer Eye Institute

University of Miami Miller School of Medicine

900 NW 17th Street, 450N, Miami, FL 33136 (USA)

E-Mail t.chang@med.miami.edu 
Fig. 1. a Slit lamp photograph of a perilimbal serpentine subconjunctival lesion. b Intraoperative gonioscopy showing open angle with moderate pigmentation.
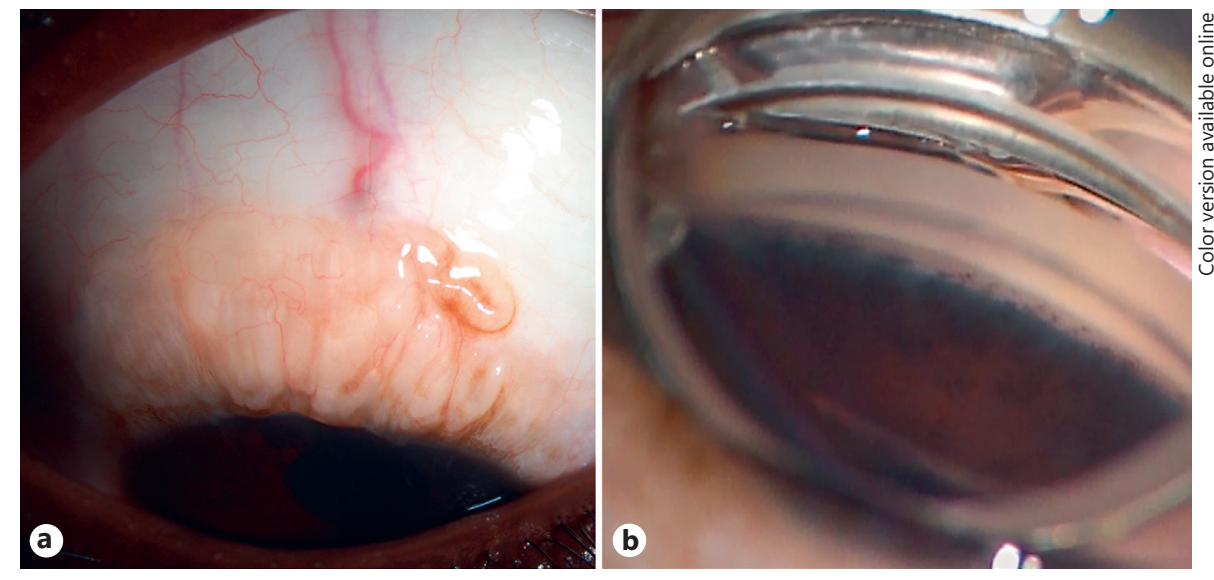

neuromas of the conjunctiva, glaucoma, and dry eyes [1, $2]$. We report the high-resolution optical coherence tomographic (HR-OCT) features and clinicopathological correlation of perilimbal mucosal neuroma in a case of pediatric MEN 2B, and the treatment of the associated secondary open-angle glaucoma with gonioscopy-assisted transluminal trabeculotomy (GATT).

\section{Case Report}

A 15-year-old girl with a history of metastatic medullary thyroid cancer was referred to the Bascom Palmer Eye Institute Emergency Room, after elevated intraocular pressures (IOPs) were noted during a routine eye examination by a pediatric ophthalmologist. A molecular diagnosis of MEN 2B syndrome was made 2 years prior with a pMet918Thr pathogenic mutation in the RET gene. There is no family history of glaucomatous diseases, and the patient has never received systemic or topical steroids. On examination, she had a bestcorrected visual acuity (BCVA) of 20/100 in both eyes (OU), constricted visual field by confrontation, and sluggishly reactive pupils. IOP by Tonopen was $28 \mathrm{~mm} \mathrm{Hg}$ in the right eye (OD) and $29 \mathrm{~mm}$ $\mathrm{Hg}$ in the left eye (OS). She was discharged on dorzolamide/timololfixed combination (OU, twice daily) and latanoprost (OU, once at bedtime), and urgently referred to the pediatric glaucoma service.

The patient was lost to follow-up and returned 20 months later. On follow-up examination, BCVA were 20/200 OD and 20/100 OS. IOPs by Goldmann applanation were $52 \mathrm{~mm} \mathrm{Hg} \mathrm{OD}$ and 44 $\mathrm{mm} \mathrm{Hg}$ OS on dorzolamide/timolol-fixed combination (OU, twice daily) and latanoprost (OU, once at bedtime). Central corneal thicknesses were $632 \mu \mathrm{m}$ OD, $616 \mu \mathrm{m}$ OS. On slit lamp examination, she had multiple, elevated, serpentine, perilimbal subconjunctival lesions (Fig. 1a) and prominent corneal nerves, but otherwise normal anterior segments in both eyes. Gonioscopy revealed Shaffer grade IV open angles with moderate pigmentation (Fig. 1b). Dilated examination revealed significant cupping and pallor of the optic discs. Color slit lamp photos and HR-OCT of the perilimbal masses were obtained (Fig. 2a), which revealed normal epithelial thickness and reflectivity, and subepithelial lesions of mixed reflectivity with lobular and whirling cellular features.

AS-OCT Imaging of Mucosal Neuromas and Treatment of Glaucoma in MEN 2B
Due to the poorly controlled IOP, GATT was performed sequentially in both eyes with concurrent excisional biopsies of the perilimbal lesions. Pathological examination revealed neural tumors composed of spindle-shaped Schwann cells (Fig. 2b) that stained with epithelial membrane antigen (Fig. 2c) and S100 protein (Fig. 2d), consistent with benign mucosal neuroma.

Three months following GATT in the second eye, BCVA has improved to 20/150 OD and 20/60 OS, and IOPs were 11 and 12 $\mathrm{mm} \mathrm{Hg}$ without the use of IOP-lowering medications. The neuromas have not recurred.

\section{Discussion}

HR-OCT is a noninvasive, adjunctive tool in the diagnosis of corneal and conjunctival pathology [3]. To our knowledge, we present the first correlation between the HR-OCT and histopathologic findings of a mucosal neuroma. The HR-OCT features of normal epithelium with subepithelial and whirling, lobular masses of mixed reflectivity differ from that of ocular surface squamous neoplasm, pingueculum, pterygium, lymphoma, Salzmann's nodular degeneration, nevi, and melanoma [3] and may help differentiate a mucosal neuroma from these other entities.

Glaucoma associated with MEN 2B has been reported in both adults and children [2, 4]. GATT, a microincisional trabeculotomy technique that allows the circumferential incision of trabecular meshwork through two clear-corneal incisions, has demonstrated fair short-term safety and efficacy in juvenile and secondary open-angle glaucoma [5]. Our patient has responded favorably to GATT in both eyes, with IOP controlled without the use of IOP-lowering medication 3 months following the procedure. Longer follow-up is needed to assess the longterm efficacy of GATT in juvenile-onset glaucoma secondary to MEN 2B. 

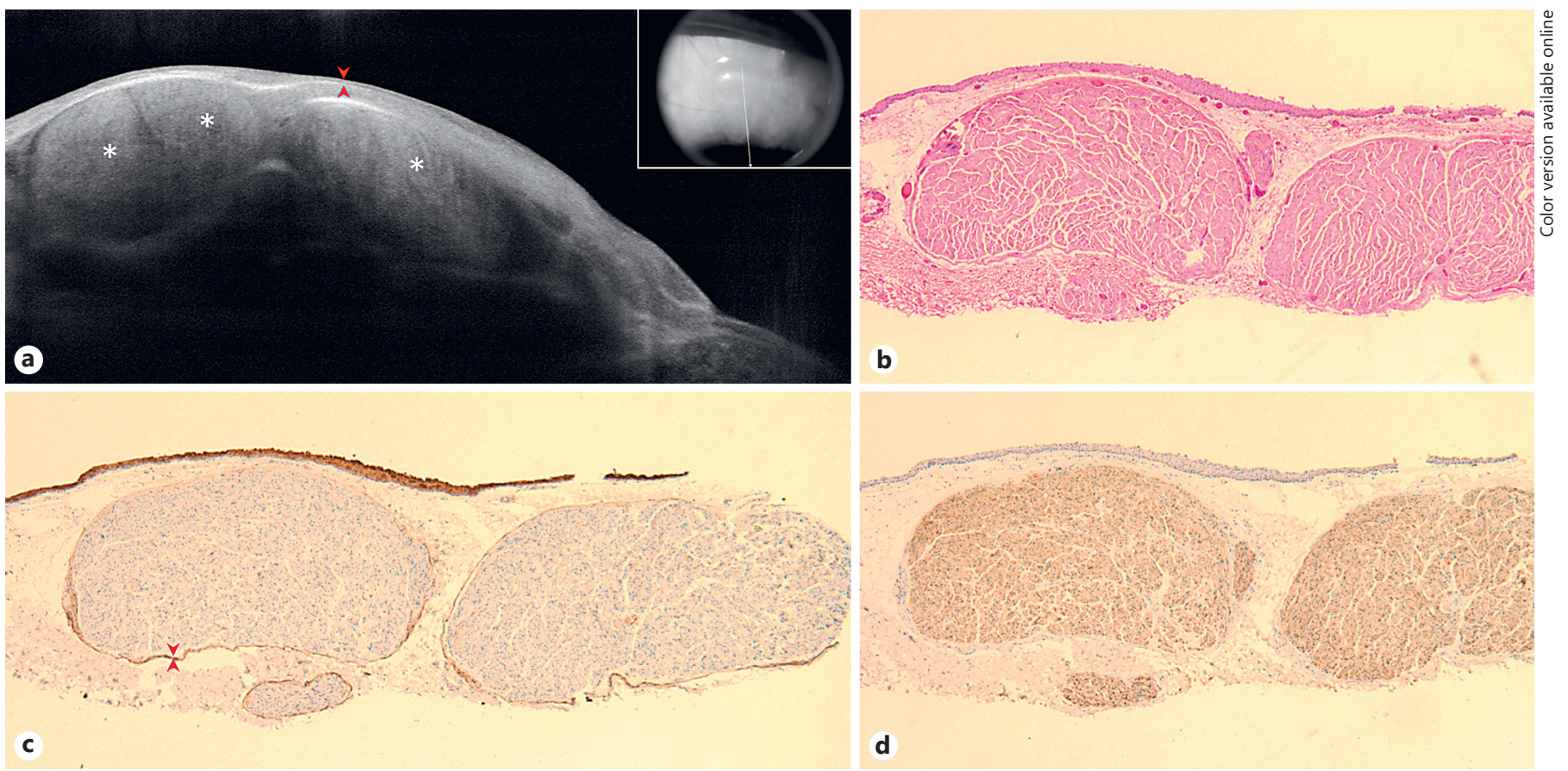

Fig. 2. a High-resolution anterior segment optical coherence tomography of the perilimbal serpentine subconjunctival lesion. The epithelium (red arrowheads) has normal thickness. There are subepithelial lobular areas of mixed reflectivity (asterisks). b Hematoxylin and eosin stain showing spindle-shaped cells (inset) that are variably encapsulated. c Positive epithelial membrane antigen stain at the tumor periphery (red arrowheads). d Positive S100 stain within the substance of the tumor. The constellation of histopathologic findings is consistent with benign mucosal neuroma.
In conclusion, HR-OCT may be a useful tool in the confirmation of conjunctival mucosal neuromas in patients with MEN 2B. GATT may be a safe and effective surgical option in children with glaucoma associated with this condition.

\section{Acknowledgement}

This study was supported by the NIH Center Core Grant P30EY014801, RPB Unrestricted Award and Career Development Awards,DepartmentofDefense(DODGrant\#W81XWH-09-1-0675), The Ronald and Alicia Lepke Grant, The Lee and Claire Hager Grant, The Jimmy and Gaye Bryan Grant, The H. Scott Huizenga Grant, The Grant and Diana Stanton-Thornbrough, The Robert Baer Family Grant, The Emilyn Page and Mark Feldberg Grant, and The Gordon Charitable Foundation and the Richard Azar Family Grant (institutional grants).

\section{Statement of Ethics}

Subject and guardian have given their informed consent for the use of clinical and pathology photographs.

\section{Disclosure Statement}

None of the authors have any possible conflicts of interest to disclose.

\section{References}

1 Jacobs JM, Hawes MJ: From eyelid bumps to thyroid lumps: report of a MEN type IIb family and review of the literature. Ophthal Plast Reconstr Surg 2001;17:195-201.

2 Beylerian M, Hoffart L, Denis D: Bilateral corneal nerve hypertrophy associated with glaucoma in a boy with multiple endocrine neoplasia type 2B. J Fr Ophtalmol 2017;40:e149-e151.

3 Nanji AA, Sayyad FE, Galor A, Dubovy S, Karp CL: High-resolution optical coherence tomography as an adjunctive tool in the diagnosis of corneal and conjunctival pathology. Ocul Surf 2015;13:226-235.

4 Mashima Y, Konishi M, Yamada M, Imamura Y, Nii S, Nakamura Y: Multiple endocrine neoplasia $2 \mathrm{~B}$ with glaucoma associated with codon 918 mutation of the RET proto-oncogene. Acta Ophthalmol Scand 1998;76:114-116.

5 Grover DS, Godfrey DG, Smith O, Feuer WJ Montes de Oca I, Fellman RL: Gonioscopy-assisted transluminal trabeculotomy, ab interno trabeculotomy: technique report and preliminary results. Ophthalmology 2014;121:855-861. 